\title{
Twenty-five years of implantable defibrillator practice in the USA: Are we ready for globalization?
}

\author{
Sanjeev Saksena
}

Published online: 29 January 2011

(C) Springer Science+Business Media, LLC 2011

\section{"Furious activity is no substitute for understanding."} H. H. Williams

As we reflect on the passage of a year and what lies ahead, few issues have occupied this discipline's attention as the continuing and riveting saga of the implantable cardioverter-defibrillator (ICD). Progressing from an investigational curiosity to mainline clinical therapy for cardiac rhythm management has been a landmark achievement. With rapid globalization in medical advances, ICD therapy now has health care implications worldwide and has become a staple item in the health care news basket.

Twenty-five years have elapsed since the initial approval of the implantable cardioverter-defibrillator for clinical use in the USA. While a quarter century of clinical experience may not qualify for the longest view of a medical therapy in the information age, there is a plethora of accumulated data and a widespread perception of a "matured" therapy. Approved for clinical use on observational clinical data, controversy dogged the early ICD years on its therapeutic value. Standards for clinical trials emerged after approval, which led to the golden age of controlled ICD clinical studies $[1,2]$.

\footnotetext{
S. Saksena $(\square)$

Electrophysiology Research Foundation, 161 Washington Valley Road, Suite 201, Warren, NJ 07059, USA

e-mail: EPRF@aol.com

S. Saksena

UMDNJ-Robert Wood Johnson Medical School,

New Brunswick, NJ, USA
}

The initial indications for ICD usage emerged largely from expert consensus documents, but have now been replaced by evidence-based practice guidelines, which, in significant part, are based on these trial data, and have seriously expanded ICD indications [3, 4]. Despite apparently robust clinical trial evidence, clinical usage patterns and therapeutic benefits have been repeatedly questioned [5]. When prophylactic ICD use became ubiquitous, this commentator was no exception, raising concerns of nonuniform benefit of prophylactic ICDs across target subgroups in the pages of this journal [6]. Since prophylactic device use now widely exceeds any other application, the potential risks to perceived ICD value in the Rosetta stone of clinical practice were even then recognizable [7]. A very high proportion of ICDs had not delivered therapy at replacement and, when used, often targeted ancillary tachyarrhythmias [5, 6]. In the MADIT 2 study, the annual rate of appropriate ICD interventions was a healthy $17 \%$, but declined to a paltry $5 \%$ when indications were widened to non-ischemic heart diseases and the ejection fraction limit raised to $40 \%$ in the Sudden Cardiac Death in Heart Failure Trial (SCD-HeFT) $[8,9]$.

The establishment of a national device registry in the United States has now bought an immense amount of clinical practice data into the fray. It has particularly highlighted discordance with clinical trial populations. At last report, over 550,000 implants (78\% for prophylactic indications), provided data showing an older ICD implant population in clinical practice with a significant octogenarian component, with fewer males, more diabetics, and hypertensives and with a predominant Caucasian background [10]. Twenty-two percent did not meet current indications, but little detail exists in registry data to identify other clinical 
imperatives that may have influenced clinical decision making [11]. For example, ICD insertion is not recommended immediately after any coronary revascularization procedures; however, the effectiveness and impact of modest regional revascularization on a large fixed arrhythmogenic substrate has been long questioned for ventricular tachyarrhythmia suppression. Similarly, ICD insertion after acute myocardial infarction is not recommended clinical practice, but sudden death in the first 3 months after myocardial infarction continues to plague the clinician in the percutaneous coronary intervention era. Simultaneously with these trends, poor penetration of ICD therapy in important subpopulations is being highlighted. Utilizing hospital discharge data from over 200 institutions and current practice guidelines, women and African American patients were noted to be less likely to be considered for or recommended potentially indicated ICD therapy [12].

It would be tempting to assume based on the preceding discussion that ICD therapy remains in an unbridled growth phase. In fact, such predictions were rather common at the beginning of the last decade and through much of it. Interestingly, recent reports of ICD utilization from 2005 to 2008 actually show a significant plateau and even decline in new implant rates, with some of the shortfall being bridged by replacement devices [13]. Heart failure ICDs constitute a solid $40 \%$ of implants, and fewer candidates for ICD prophylaxis are being recruited nationwide. While this may not necessarily be surprising, assuming that the prevalence pool is being addressed and national sudden death rates have been on the decline, the absolute mismatch in numbers remains intriguing.

These data can indeed be used to fuel efforts at increasing accurate identification and equitable access to ICD therapy. However, even available risk stratification methods using commonly available investigations in a composite scheme, or even standard electrophysiologic testing when applied in appropriate subgroups that can improve accuracy in identification of high and low risk individuals, have not been widely accepted or implemented $[14,15]$. Furthermore, efforts to identify the broad spectrum of potential sudden death victims in the overall population have continued to languish. The desire for powerful and absolute specific marker(s) of sudden death risk has ignored the increasingly multi-factorial genesis of sudden cardiac death. New approaches such as use of implantable loop recorders post infarction have had limited traction despite its potential value [16]. While attention has focused on sudden death in high-risk cardiac patients, the equivalent number of sudden deaths in lower-risk populations has received scant clinical attention. Markers such as overall ischemic burden in coronary populations without significant left ventricular dysfunction have only recently been seriously studied [17].

So, where does ICD therapy go from here? With worldwide dissemination of ICD therapy in progress, the US experience is key to deploying ICDs more effectively globally. While the US health care system has absorbed the costs of ICD access, the overall impact of the ICD on sudden death in the USA is still awaited. We expect epidemiologic data to be forthcoming shortly. This will be key in balancing concerns regarding less than optimally focused application of this therapy and health care resource consumption. Our focus then should shift to the practice guideline process and better patient selection from highand lower-risk prevalence pools. Having moved away from expert consensus, the evidence-based guideline process is constructed by the emergence of clinical trial data. The prioritization and design of these trials are independent of health care planning implications and investigator-driven. For example, stringent risk stratification in SCD-HeFT trial was suggested and rejected (personal communication). It is now increasingly clear that we need a course correction in our practice guideline process. The rebalancing of the practice guideline process now needs to consider trial design elements, critically review subgroup risk distribution and effectiveness of therapy, and achieve expert consensus on the best indications and incremental value. Ignoring risk stratification schemes is no longer plausible or economically tenable. The additive value of a new therapy can have variable outcomes that need to be balanced with cost implications [18]. Many nations, such as the United Kingdom, have institutions that approve introduction of new therapies, in such a manner [19].

For global value to ICD therapy, the lessons learned from the US experience are both invaluable and thought provoking [20]. While there can be little debate around ICD therapy for secondary prevention, the primary prevention usage of this device is still in evolution. The ICD experience positively challenges the practice guideline and technology evaluation processes to evolve to higher levels of value to both patients and health care systems. In doing this, it serves as an important model for technology absorption in health care systems and will positively influence technology development.

2010 was another year of continued solid growth for the journal, and 2011 brings brand-new opportunities. We owe our continued success in great measure to the unflinching efforts of our authors, reviewers, editorial board, and journal staff. The editors have risen and responded to the rapid changes in the world of medical publications and I thank them, once again, for their continuing investment in the Journal. 


\section{References}

1. Saksena, S., Camm, A. J., Bilitch, M., Fisher, J. D., Furman, S., Griffin, J. C., et al. (1987). Clinical investigation of implantable antitachycardia devices: report of the Policy Conference of the North American Society of Pacing and Electrophysiology. Journal of the American College of Cardiology, 10, 225-229.

2. Saksena, S., Epstein, A. E., Lazzara, R., Maloney, J. D., Zipes, D. P., Benditt, D. G., et al. (1995). Report of the policy conference on clinical investigation of antiarrhythmic devices. A position statement of the North American Society of Pacing and Electrophysiology, American College of Cardiology, American Heart Association, and Working Groups on Arrhythmias and Cardiac Pacing of the European Society of Cardiology. Circulation, 91, 2097-2109.

3. Lehmann, M. H., Saksena, S., \& the NASPE Policy Conference Committee. (1991). Implantable cardioverter defibrillators in cardiovascular practice: report of the Policy Conference of the North American Society of Pacing and Electrophysiology. Pacing and Clinical Electrophysiology, 14(6), 969-979.

4. Epstein, A. E., DiMarco, J. P., Ellenbogen, K. A., Estes, N. A., 3rd, Freedman, R. A., Gettes, L. S., et al. (2008). Guidelines for device-based therapy of cardiac rhythm abnormalities: a report of the American College of Cardiology/American Heart Association Task Force on Practice Guidelines (Writing Committee to Revise the ACC/AHA/NASPE 2002 Guideline Update for Implantation of Cardiac Pacemakers and Antiarrhythmia Devices) developed in collaboration with the American Association for Thoracic Surgery and Society of Thoracic Surgeons. Journal of the American College of Cardiology, 51(21), e1-e62.

5. Tung, R., Zimetbaum, P., \& Josephson, M. E. (2008). A critical appraisal of implantable cardioverter-defibrillator therapy for the prevention of sudden cardiac death. Journal of the American College of Cardiology, 52(14), 1111-1121.

6. Saksena, S. (2005). Federal guidelines for prophylactic ICD therapy in high risk populations for sudden cardiac death: is this a necessary course correction? Journal of Interventional Cardiac Electrophysiology, 12(1), 5-7.

7. Saksena, S. (2005). Prophylactic ICDs: can (and will) the medical marketplace decide their role? Journal of Interventional Cardiac Electrophysiology, 12(3), 175-176.

8. Singh, J. P., Hall, W. J., McNitt, S., et al. (2005). Factors influencing appropriate firing of the implanted defibrillator for ventricular tachycardia/fibrillation: findings from the Multicenter
Automatic Defibrillator Implantation Trial II (MADIT-II). Journal of the American College of Cardiology, 46, 1712-1720.

9. Bardy, G. H., Lee, K. L., Mark, D. B., et al. (2005). Amiodarone or an implantable cardioverterdefibrillator for congestive heart failure. New England Journal of Medicine, 352, 225-237.

10. Hammill, S. C., Kremers, M. S., Kadish, A. H., et al. (2009). Review of the ICD Registry's third year, expansion to include lead data and pediatric ICD procedures, and role for measuring performance. Heart Rhythm, 6, 1397-1401.

11. Hernandez, A. F., Fonarow, G. C., Liang, L., Al-Khatib, S. M., Curtis, L. H., LaBresh, K. A., et al. (2007). Sex and racial differences in the use of implantable cardioverter-defibrillators among patients hospitalized with heart failure. JAMA, 298(13), $1525-1532$.

12. Al-Khatib, S. M., Hellkamp, A., Curtis, J., Mark, D., Peterson, E., Sanders, G. D., et al. (2011). Non-evidence-based ICD implantations in the United States. JAMA, 305(1), 43-49.

13. Nudell, B. (2009) UBS investment report, December 7, 2009

14. Kearney, M. T., Fox, K. A., Lee, A. J., Brooksby, W. P., Shah, A. M., Flapan, A., et al. (2004). Predicting sudden death in patients with mild to moderate chronic heart failure. Heart, 90(10), 1137-1143.

15. Iesaka, Y., Nogami, A., Aonuma, K., Nitta, J., Chun, Y. H., Fujiwara, H., et al. (1990). Prognostic significance of sustained monomorphic ventricular tachycardia induced by programmed ventricular stimulation using up to triple extrastimuli in survivors of acute myocardial infarction. The American Journal of Cardiology, 65(16), 1057-1063.

16. Huikuri, H. V., Raatikainen, M. J., Moerch-Joergensen, R., Hartikainen, J., Virtanen, V., Boland, J., et al. (2009). Prediction of fatal or near-fatal cardiac arrhythmia events in patients with depressed left ventricular function after an acute myocardial infarction. European Heart Journal, 30(6), 689-698.

17. Piccini, J. P., Starr, A. Z., Horton, J. R., Shaw, L. K., Lee, K. L., Al-Khatib, S. M., et al. (2010). Single-photon emission computed tomography myocardial perfusion imaging and the risk of sudden cardiac death in patients with coronary disease and left ventricular ejection fraction $>35 \%$. Journal of the American College of Cardiology, 56(3), 206-214.

18. Califf, R. M., \& DeMets, D. L. (2002). Principles from clinical trials relevant to clinical practice: part I. Circulation, 106(8), 1015-1021.

19. National Institute for Health and Clinical Excellence: http:// guidance.nice.org.uk/TA95/Guidance.

20. Saksena, S. (2006). Achieving global access to heart rhythm therapies in the next decade: a tangible goal. Journal of Interventional Cardiac Electrophysiology, 17, 163-168. 